\title{
Spontaneous rupture of the oesophagus with unusual features
}

\author{
MAJOR D. M. ROBERTS \\ M.B.(Lond.), M.R.C.P.E., R.A.M.C. \\ Senior Specialist in Medicine
}

\author{
Major D. O. H. Messent \\ M.B.(Syd.), F.R.C.S., R.A.M.C.
}

Senior Specialist in Surgery

British Military Hospital, Munster, B.F.P.O. 17

SPONTANEOUS rupture of the oesophagus is an uncommon but not a rare condition. Early diagnosis is essential because the patient's only hope for survival lies in early definitive surgery. Even with surgery, the survival rate varies between only 64\% (Derbes \& Mitchell, 1956) and 73\% (Anderson, 1952). Without surgery death is inevitable (Derbes \& Mitchell, 1956).

The diagnosis is often not difficult, provided the condition is borne in mind, although it has, on occasion, been confused with many other conditions, including perforated peptic ulcer, myocardial infarction, acute pancreatitis, dissecting aneurysm of the aorta, spontaneous pneumothorax and strangulated internal hernia (Ware et al., 1952; Derbes \& Mitchell, 1956).

We describe here a case of spontaneous rupture of the oesophagus in which some extraordinary features occurred, confusing the diagnosis.

\section{Case report}

On the morning after an evening's drinking, a 66-year-old retired soldier felt unwell and vomited. Whilst vomiting a second time, he was struck down by a sudden severe pain spreading around his upper abdomen. The pain was excruciating, and he gained most relief from being propped up in a sitting position. Although breathing was painful, he was not unduly short of breath. He was immediately admitted to hospital. He had previously been a fit man, apart from occasional mild heartburn after meals.

On admission, he was restless, but not unduly shocked. The temperature was $101 \cdot 5^{\circ} \mathrm{F}\left(38 \cdot 6^{\circ} \mathrm{C}\right)$. There were no abnormal signs in the cardiovascular system or the chest. There was generalized abdominal tenderness and guarding, progressing rapidly to four-quadrant board-like rigidity, although bowel sounds were present. Blood picture, serum amylase and plain $\mathbf{X}$-ray of the abdomen were all normal. Although it was appreciated that there were atypical features, the most likely diagnosis was thought to be perforated peptic ulcer and, accordingly, $4 \frac{3}{4} \mathrm{hr}$ post-ictus, laparotomy was carried out. No abnormality was found.
On recovering from the anaesthetic, he was very ill indeed; he remained in severe pain, and respirations were short and grunting. A few crepitations were heard at the right lung base and a very small area of subcutaneous emphysema was found above the right clavicle. Serum transaminases were normal, and an electrozardiogram showed right bundle branch block. He was nursed in an oxygen tent and given full antibiotic cover.

By the next morning there were impairment of percussion note, diminished breath sounds and crepitations at both lung bases, and the subcutaneous emphysema had, significantly, spread to involve both sides of his neck. A chest X-ray revealed fluid in the left pleural cavity, sligh increased shadowing at the right lung base, an slight widening of the mediastinum. These de velopments immediately suggested a ruptured. oesophagus. Aspiration of the left pleural cavity was unsuccessfully attempted. The patient, however, was considered too ill for further investigation or surgery at this stage.

On the 4th day of his illness, slight but distinct improvement in his general condition occurred, and contrast medium $\mathrm{X}$-ray studies were undertaken. Screening revealed the medium leaving the lower end of the Levin's tube which was, unexpectedly, above the diaphragm, entering a cavity and forming a fluid level (see Figs. 1 and 2). This cavity was taken to be a partially herniated, obstructed stomach. Below the left diaphragm was another cavity, thought to be the antral part of the stomach, into which a small amount of the contrast medium was seen to pass. At this stage, with what appeared to be a viscus in the thorax, the diagnosis was rapidly revised. It was felt that the explanation most likely to fit the clinical and radiological features was an acute partial herniation of the stomach into the thorax, with initial obstruction and strangulation, now beginning to settle, with partial relief of the obstruction.

Thereafter, he was given small amounts of fluid by mouth, and this was gradually increased until, by the 9th day, he was taking sufficient 


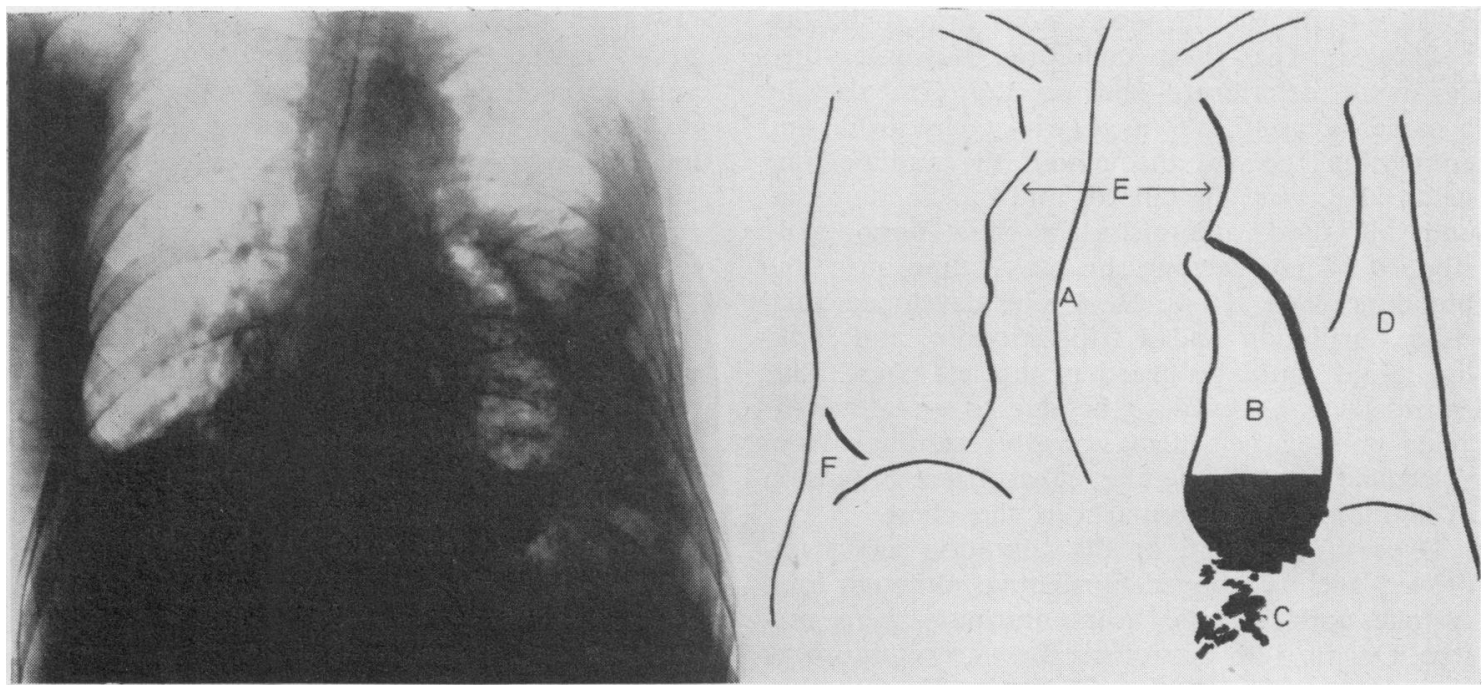

FIG. 1. Chest X-ray and tracing showing the pyopneumothorax simulating a partially herniated, partially obstructed stomach within the chest. Contrast medium has been instilled through the Levin's tube. A, The Levin's tube in the oesophagus, deviated to the right; B, cavity of the pyopneumothorax, which was mistaken for stomach, a fluid level is well shown; C, some contrast medium apparently passing through the diaphragm; D, fluid within the left pleural cavity; $\mathrm{E}$, widened mediastinum; $\mathrm{F}$, small amount of fluid in the right pleural cavity.

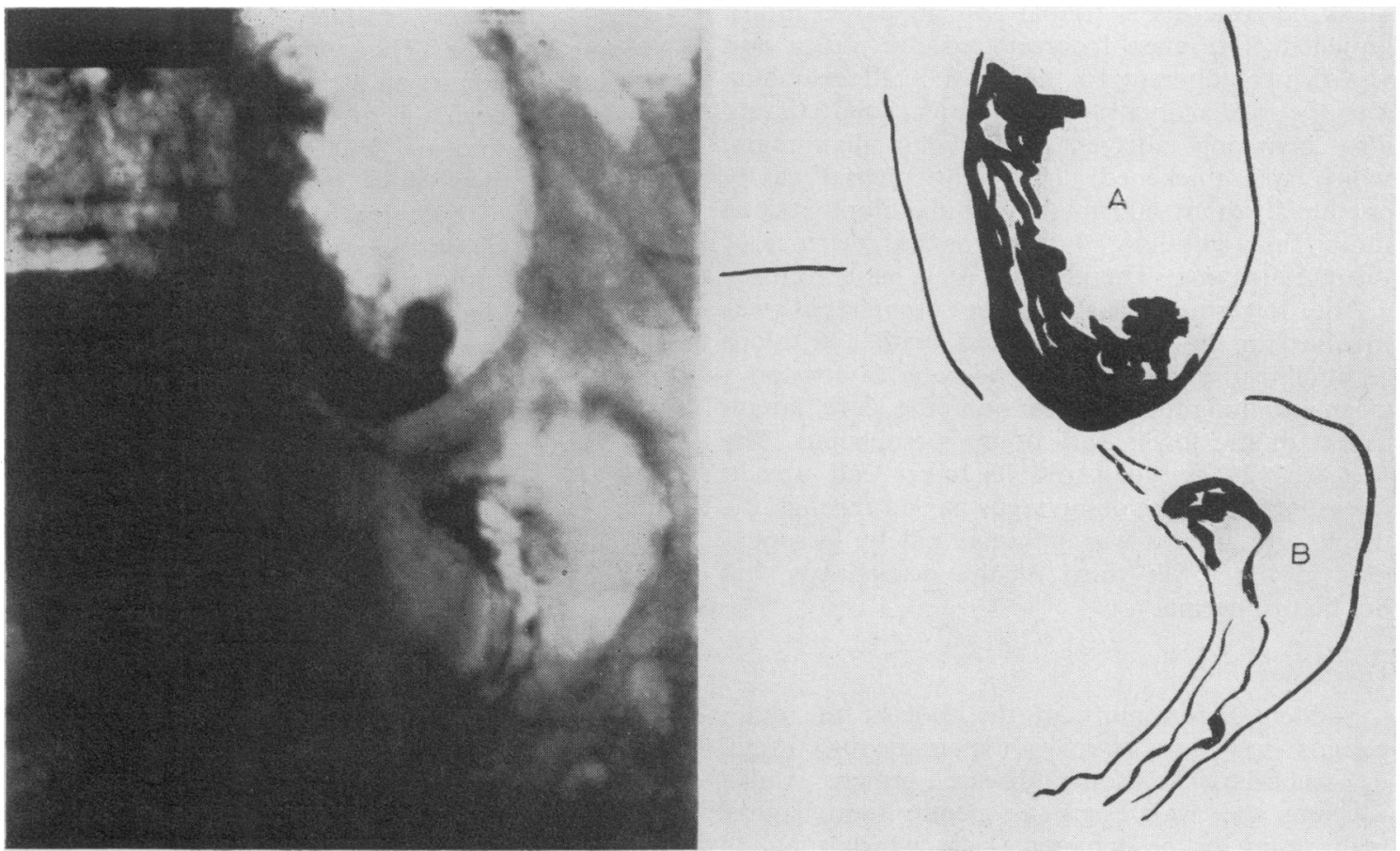

FIG. 2. X-ray and tracing showing an oblique view taken during screening, using contrast medium. A, cavity of the pyopneumothorax, simulating the herniated, upper portion of a stomach above the diaphragm; B, actual stomach, but taken to be only a portion of the organ remaining below the diaphragm. 
by this route for the intravenous drip to be discontinued. The chest condition, however, then began to deteriorate and on the 11 th day he became intensely dyspnoeic, with tachycardia, and crepitations present throughout the audible lung fields. He was digitalized and given diuretics, and all fluid administration was temporarily stopped. That evening he was stuporous; the blood pressure fell to $80 / 45$, he developed auricular fibrillation and a triple rhythm, and tinkling' fluid could be heard in the left chest. This episode was believed to be due to an attack of acute pulmonary oedema, possibly resulting from a cardiac tamponade-like effect from distension of the obstructed stomach in the chest.

Over the next $36 \mathrm{hr}$ his condition again improved and he stopped fibrillating, although loud systolic and diastolic heart murmurs were present. On the 12th day of his illness a jejunostomy was done under local anaesthesia. Despite this, deterioration was thereafter progressive. Terminally, he developed a pericardial friction rub and finally slipped into coma and died 14 days and $8 \mathrm{hr}$ after the onset of his illness.

Necropsy. Apart from the effects of surgery, changes of significance were confined to the thorax. The whole of the left lung, except for a small part at the apex, consisted of a greyishblack necrotic mass, friable and, in parts, almost liquefied. The mass incorporated the pleura and was firmly adherent to the chest wall, extending into the mediastinum, where it was cavitated, and also involving adjacent underlying diaphragm, which was thickened. The right pleural cavity contained about $600 \mathrm{ml}$ of slightly blood-stained fluid. The right lung showed marked oedema of the parenchyma. There was a dry pericarditis.

The lowermost part of the oesophagus was involved in the necrotic process, with a fistulous communication between the necrotic cavity and a clean-cut longitudinal tear on the left lateral aspect of the lower part of the oesophagus. The tear was $2 \frac{1}{2} \mathrm{~cm}$ long and its lower end was at the point where the oesophagus passed through the diaphragm. There was minimal patchy oesophagitis in the lower third of the oesophagus, but no hiatus hernia.

\section{Discussion}

Sudden discontinuity of the wall of the oesophagus may be a primary ('spontaneous') event, or secondary to some disease process which weakens the wall, such as peptic oesophagitis, malignancy or oesophagomalacia which may, in turn, be secondary to a cerebral lesion (Maciver et al., 1956). Our case is one of spontaneous rupture and conforms to the definition of Mackler
(1952) by differing from other forms in two res-

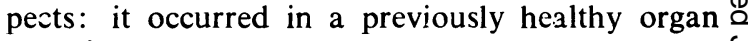
and, in contrast to the small round hole of a $c$ perforation, the rupture appeared as a clean-cut $\overrightarrow{\bar{N}}$ longitudinal rent at or near the oesophago-gastric junction. 'Spontaneous' is, perhaps, an inappropriate word because, as Tesler \& Eisenberg (1963) write in their excellent review of the subject: 'The most consistent historical event in spontaneous oesophageal rupture is the act of vomit- os ing'. A few cases have been reported of this $\vec{O}$ catastrophe being the result of other causes of $\overrightarrow{\vec{\omega}}$ explosive increase in intra-abdominal pressure $\omega_{\mathcal{O}}$ (Mackler, 1952 ; Aldrich \& Anspach, 1939; Klein \& Grossman, 1943 ; Kennard, 1950), or of pneumatic trauma (Kerr et al., 1953).

Many of the presenting features were typical of ruptured oesophagus (Barrett, 1946; Ware et $\stackrel{\omega}{\omega}$ al., 1952; Tesler \& Eisenberg, 1963). The onset $\dot{\sigma}$ of pain during vomiting but with no recurrence $\vec{A}$ of vomiting thereafter is very suggestive, as was 음 the nature of the pain itself, and the fact that he gained most relief from being propped up in $\mathbb{D}$ a sitting position. The triad of signs which should suggest the diagnosis when present (Barrett, 1947) - rapid respiration, upper abdominal rigidity and subcutaneous emphysema-was incomplete in the early stages. There was little evidence of per pheral circulatory failure, which is usually Q striking feature. The temperature was slightly elevated on admission, whereas it is usually normal or subnormal in the first few hours after rupture of the oesophagus. But the one feature, more than any other, which we considered to indicate a primarily abdominal lesion was the rapidly developing board-like rigidity in all four quadrants of the abdomen. As far as we are aware, $\supset$ this feature has been reported only once before in ruptured oesophagus (Tidman \& John, 1967). 흔 The majority of cases do show rigidity and tenderness in the upper abdomen, but examination of the lower abdomen is usually negative. In any case, perforated peptic ulcer is the most common misdiagnosis, even though there is usually an absence of peptic ulcer history (Ware et al., 1952). 을

The earliest and most consistent features related $\frac{D}{O}$ to the intrathoracic pathology are usually dyspnoea, orthopnoea and cyanosis, followed by $\mathcal{O}^{\circ}$ pleural effusion, usually on the left side, and $N$ eventually empyema. A 'mediastinal crunch' o (Hamman's sign), may be heard at any stage. $\omega$ During the first few hours of admission this patient exhibited no abnormal signs in the chest. Despite this, the suggestive sequence of events $\stackrel{D}{\oplus}$ which is usual as the disease progresses, namely the abdominal signs decreasing in magnitude in contrast to the increasing thoracic signs, did $\mathbb{D}$ 
occur, although the abdominal features, of course, were modified by the laparotomy.

The diagnosis, then, was thought to be 'settled' by the appearance not only of signs in the chest but of increasing subcutaneous emphysema about the neck. Although subcutaneous emphysema may, rarely, be a presenting sign, it is more commonly delayed. It is very highly suggestive of ruptured oesophagus, occuring in about $65 \%$ of cases (Tesler \& Eisenberg, 1963), but is not conclusive. It has been suggested that it appears early unless there is early rupture of the pleura, and that is presumably what happened in this patient.

In retrospect it can be seen that this man's only hope of survival, even at that stage, lay in further definitive surgery. This incontestable fact is well demonstrated by a reported series of seventy-one cases of ruptured oesophagus in which surgery was not attempted; all were dead within a week (Derbes \& Mitchell, 1956). We felt that a second major operation at any stage after the first would have killed the patient forthwith and, although it has been suggested (Tesler \& Eisenberg, 1963) that even a moribund state should not constitute a contraindication to surgery, few surgeons, perhaps, would gainsay our reluctance.

Radiological findings in ruptured oesophagus are reviewed by Priviteri \& Gay (1951). Very few cases show no radiological change. Hydrothorax, hydropneumothorax or pneumothorax is usual, such changes being more frequent on the left side than the right. Cervical and mediastinal emphysema are common; the mediastinum is occasionally shifted and sometimes actually widened. Lynch (1949) has commented on the presence of a retrocardiac air bubble with a fluid level. Mediastinal emphysema has rarely been reported following perforated gastric (Welty, 1949) or duodenal ulcer (McCorkie \& Stevenson, 1937). The early radiological features in this patient were, therefore, strongly in favour of the diagnosis of ruptured oesophagus.

Demonstration of the fistula by contrast medium is diagnostic and also serves to locate the site of the tear (Priviteri \& Gay, 1951). Water-soluble contrast material may be instilled through a Levin tube (Christofordis \& Nelson, 1957), and we undertook Gastrografin studies just as soon as we judged the patient able to withstand the procedure. The findings surprised us. The appearances realistically simulated the presence of about two-thirds of the stomach in the chest. Taking this at its face value we felt we could but postulate that sudden herniation of the major portion of the stomach through the diaphragm had resulted in obstruction and strangulation of that organ, these subsequently becoming partly relieved. The radiological appearances were so realistic that we were forced to discount the negative laparotomy findings. As far as we know, this deceptive radiological picture has not been reported before, although Tesler \& Eisenberg (1963) remark, in connection with pleural aspiration after ruptured oesophagus, that "care must be taken to ascertain that the needle is not in a dilated, herniated stomach'. It was, of course, the loculated pyopneumothorax in fistulous communication with the oesophagus which we were seeing, but the position, shape and size were identical with a herniated stomach, and the irregular walls of the cavity even gave rise to an appearance suggestive of coarse gastric mucosal folds.

From that moment our hope was that relief of the 'obstruction' would become complete and we did, in fact, find that for a few days he could tolerate increasing quantities of fluid by mouth. When he next relapsed, with intense dyspnoea due to pulmonary oedema, we postulated a cardiac tamponade-like effect due to distension of what was thought to be the stomach. Thereafter, out intention was to do a jejunostomy at the earliest feasible opportunity, leaving any possible definitive abdomino-thoracic surgery until a much later stage. Acute pulmonary oedema following ruptured oesophagus has been reported only once before, by Ellis (1956). In his case, there was also asymmetry of the pulses in the arms, and Ellis suggested that both features were due to either compression or spasm of the great vessels in the mediastinum, despite the fact that no abnormality of these was found at postmortem. It seems to us that interference with diastolic filling of the heart by the pressure of fluid and air in the mediastinum is a more likely explanation.

In view of the mortality findings of Derbes \& Mitchell (1956), and their finding that over $80 \%$ of cases occur between the ages of 35 and 65 years, the fact that a man of 66 years survived, without definitive surgery, for 14 days and $8 \mathrm{hr}$ is, in itself, extraordinary.

\section{Summary}

A case of 'spontaneous' rupture of the oesophagus in a 66-year-old man is described. He presented unusually, with rapidly developing board-like rigidity of his abdomen in all four quadrants, and was, therefore, submitted to laparotomy. His subsequent progress made the correct diagnosis clear, but that diagnosis was then confounded by an extraordinary radiological appearance: on instilling a contrast medium into the 
oesophagus, some entered the cavity of a loculated pyopneumothorax, closely simulating the appearance of a partially herniated, partially obstructed stomach within the chest. Without definitive surgery, he survived for more than 14 days.

\section{Acknowledgments}

We thank Brigadier P. R. Wheatley, D.s.o., F.R.C.s., and Brigadier M. F. Kelleher, O.B.E., M.C., F.R.C.P., for permission to publish details of this patient; Colonel N. C. Rogers, F.R.C.s., and Colonel J. F. Webb, M.C., M.D., M.R.C.P.E., for advice during management of the case; Lieutenant-Colonel A. F. Young, M.B., D.M.R.D., for radiological assistance; and Colonel T. G. A. L. Warrington, M.D., for the postmortem.

\section{References}

Aldrich, C.A. \& ANSPaCh, W.E. (1939) Rupture of esophagus from a blow on the abdomen. Radiology, 32, 93.

BARRETT, N.R. (1946) Spontaneous perforation of the oesophagus; review of literature and report of three new cases. Thorax, $1,48$.

BARRETT, N.R. (1947) Report of a case of spontaneous perforation of oesophagus successfully treated by operation. Brit. J. Surg. 35, 216.

Christofordis, A. \& Nelson, S.W. (1957) Spontaneous rupture of esophagus. Amer. J. Roentgenol. 78, 574.

Derbes, V.J. \& Mitchell, R.E., JR (1956) Rupture of the esophagus. Surgery, 39, 688.
Ellis, F.G. (1956) An unusual case of rupture of the oesophagus. Guy's Hosp. Rep. 105, 229.

KeNNARD, H.W. (1950) Rupture of the oesophagus during $c$ childbirth. Brit. med. J. i, 917.

KerR, H.H., Sloan, H. \& O'Brien, C.E. (1953) Rupture ज़ of the esophagus by compressed air. Surgery, 33, 417 .

Klein, L. \& Grossman, M. (1943) Rupture of the esophagus. Med. Bull. Veterans' Adm. (Wash.), 19, 277.

LYNCH, S.P. (1949) Spontaneous perforation of the esophagus. Report of three cases successfully treated surgically. New Engl. J. Med. 241, 395.

Maciver, I.N., Smith, B.J., Tomlinson, B.E. \& Whitby, ๗ூ J.D. (1956) Rupture of the oesophagus associated with lesions of the nervous system. Brit. J. Surg. 43, 505.

MACKLER, S.A. (1952) Spontaneous rupture of the esophagus; an experimental and clinical study. Surg. Gynec. Obstet. 95, 345.

McCorkie, H. \& Stevenson, J. (1937) Subcutaneous emphysema associated with a perforated gastric ulcer. Surgery, 2, 930.

Priviteri, C.A. \& GAY, B.B., JR (1951) Spontaneous rupture of the esophagus. Radiology, 57, 48.

Tesler, M.A. \& EisenberG, M.M. (1963) Spontaneous esophageal rupture. Int. Abstr. Surg. 117, 1.

Tidman, M.K. \& JoHN, H.T. (1967) Spontaneous rupture of the oesophagus. Brit. J. Surg. 54, 286.

WARE, G.W., ShNider, B.I. \& Davis, E.W. (1952) Spontaneous rupture of esophagus. Arch. Surg. 65, 723.

Welty, D.M. (1949) Mediastinal emphysema following anterior perforation of a gastric ulcer. Ann. intern. Med. 30, 205. 